\title{
STRUCTURAL ADAPTIVE ANISOTROPIC NAS-RIF FOR BIOMEDICAL IMAGE RESTORATION
}

\author{
Paramate Horkaew*, Tanawat Kwanpak \\ Suranaree University of Technology, Thailand
}

Blind image deconvolution is an ill-posed problem that attempts to restore an acquired image degraded by unknown PSF. A variational BID implementation, called NAS-RIF, is known for being robust but prone to poor convergence under low SNR and unrealistic support. Motivated by simple yet efficient fidelity metric, this paper presents an improved NAS-RIF by reducing adverse effect of inverse high-pass filter and computationally intensive pre-deterministic noise removal, by adaptively incorporating anisotropic structural property within local neighborhood seamlessly in NAS-RIF cost function. With an automatic support region estimation, the entire deconvolution process was fully automatic. The experimental results reported herein indicated that the enhanced structural adaptive anisotropic NAS-RIF had better convergence condition, while maintaining the underlying image fidelity.

Key words: Blind Deconvolution, NAS-RIF, Structural Adaptive Filter

\section{INTRODUCTION}

Signal degradation has been considered one of the most impeding factors in computer vision and pattern recognition [1] In medical imaging, it often occurs during acquisition and reconstruction processes. Its root cause stems from non-ideal sensors (e.g., x-ray detector and ultrasonic transducer, etc.) or subject motion.Certain degradations such as contrast, noise, and blur were shown associated with diminished performance and appreciation of radiographic imagery [2]. Provided various assumptions on and extents of degradations, higher-level vision strategieshave often been incorporated to assist analyses,e.g., recognition [3], fusion [4], segmentation[5],and modeling [6], etc. However, depending on certain types of analyses, imaging fidelity, i.e., luminance, contrast, and structural appearance remains imperative to their performance, which could be undermined by ill-considered enhancements. Restoration of the "true" image involves identifying the underlying degrading model, such as noises or a Point Spread Function (PSF) and then performing the respective inverse operations (Fig 1). Among stateof-the-arts approaches, deconvolution is characterized by computing procedure that corrects an image, for the influence of instrumental PSF and possibly noises. It is based on a hypothesis that the recorded image is indeed the convolution of the actual distribution of an object with PSF. Deconvolution thus treats image as an estimate ( $\left.f^{\prime}\right)$ of the true signal intensity (f) and using an expression for the PSF (h), performs mathematical inverse ( $h$ ') of the imaging process $(\mathrm{g})$ to obtain an improved estimate of image intensity, as shown in (Fig 1)[7].

Practical pattern recognition system often relied on explicit knowledge on the PSF such as low contrast, outof-focus, and motions and then reconstruct the original scene by filtering an acquired image with predetermined linear kernel, which often was the inversed filter of a degrading model. Given a model of PSF (such as Gaussian blur), its representing parameters (e.g., standard deviation, skew, orientation, etc.) can be accurately obtained, for instance, by first acquiring a single object with known intensity and then performing a Maximum Likelihood $(\mathrm{ML})$ parametric estimation based on actual and acquired signals [8]. Blind Image Deconvolution (BID) [9, 10], on the other hand, tackles this restoration problem without knowing of such kernel nor its parameters in advance. Although BID is a mature field, it remains one of the most challenging issues in image analysis. BID is known for being an ill-posed problem, where small variation in data (e.g., imaging noise) can lead to very large variations in the solution, hence the estimated kernel and restored image [10]. Instead of optimizing model parameters, BID requires an estimation of many more unknown variables, i.e., all kernel elements, based on some metrics. It is hence considered an under-determined non-linear inverse problem and normally been tackled by iteratively learning from small observations. More recent reviews and discussions on BID methods, such as application areas, complexities, convergence properties, and other implementation issues can be found in [11, 12]. With BID [12], it is assumed that discrete image degradation is caused by the linear convolutional blur and additive noise and is expressed by:

$$
g(\mathbf{x})=\sum_{s \in S_{h}} h(\mathbf{x}-\mathbf{s}) f(\mathbf{s})+n(\mathbf{x})
$$

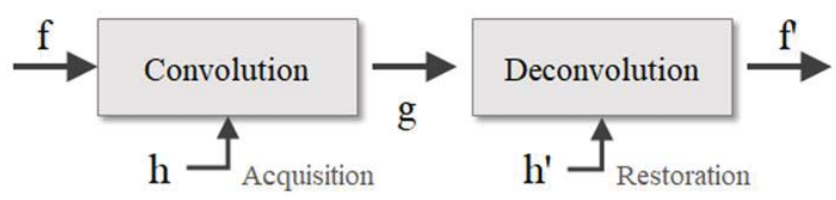

Figure 1: Overview of deconvolution process to estimate a true image, $f$ ' 
where $f(\mathrm{x}), g(\mathrm{x}), h(\mathrm{x}, \mathrm{s})$, and $n(\mathrm{x})$ represent the original image, the observed image, a space invariant blur (PSF), and observation noise, respectively. $S_{h} \subset R^{2}$ is the support of the PSF. The additive noise, $\mathrm{n}$ occurs during imaging acquisition process.

Trivial restoration could exploit the linear properties of the convolution and be achieved by using an inverse filter in the frequency domain. This operation however is known to suffer from instability due to unrealistic noise model in actual applications. Iterative Blinded Deconvolution (IBD) [13] was proposed to overcome this issue. Starting from a random PSF IBD iteratively estimates PSF in the Fourier domain, while imposing constraints on non-negativity of the pixel intensities and finites support. Similarly, the Nonnegativity and Support Constraints Recursive Filtering (NAS-RIF), proposed in [14, 15, 16], iteratively estimates the restoring PDF that has an inverse and is absolutely summable. NAS-RIF derives a cost function based on the same constraints as the IBD method, but its optimization process is carried out in the spatial domain. In NAS-RIF, these constraints are imposed by projecting the estimated image onto a convex set via POCS (Projection onto Convex Set). The method has been widely applied in medical and astronomical imaging. Although several extensions have since been attempted to improve the convergence and quality of the restored image [17-23], enhancing of NAS-RIF with state-of-art algorithms remains an open area of investigation.

This paper thus proposes a structural adaptive image restoration based on NAS-RIF algorithm. By determining local structure of an image, resultant anisotropic weights were incorporated into the NAS-RIF objective function. It would be exhibited both quantitatively and visually by the experiments herein that the resultant restoration had good stability against degradation and image quality. More specifically, the emphasis was placed on image intensity, gradient, and appearance structure, as they are pertinent to medical image analysis tasks, i.e., segmentation, edge detection, and fusion, respectively. The remaining of the paper is organized as follow. The next section discusses existing works on IBD, with emphasis on iterative NAS-RIF, and other related materials employed in this study. Next, the proposed method is explained in greater details. Subsequently,both numerical and visual experimental results are illustrated. Finally, the discussions and concluding remarks of this paperare provided.

\section{MATERIALS AND METHODS}

\section{IBD}

Following the notations in equation (1), IBD is a process of estimating the true image, $f$, and the degrading blur, $h$, from a degraded image, $g$, provided a priori on the imaging system [10, 24]. Linear image restorations iteratively determine a set of model parameters from a known blurring function via inversion, subject to particular degradation and image model. IBD simultaneously estimates both pixels of the true image and the PSF (or its inverse), by using numerical techniques with non-negativity and support constraints incorporated into its objective (cost) function. The most well-known variant of IBD was proposed by Ayers and Dainty [13], where both true image and PSF were recovered in alternate in the frequency domain, using Fast Fourier Transform (FFT). The constraints were imposed by replacing violations with predefined pixel values, with respect to region of support. At $\mathrm{k}^{\text {th }}$ iteration, PSF $(\mathrm{H})$ and true image $(\mathrm{F})$ were expressed as follow:

$$
\begin{aligned}
& \widetilde{H}_{k}(u, v)=\frac{G(u, v) \hat{F}_{k-1}^{*}(u, v)}{\left|\hat{F}_{k-1}(u, v)\right|^{2}+\alpha /\left|\widetilde{H}_{k-1}(u, v)\right|^{2}} \\
& \widetilde{F}_{k}(u, v)=\frac{G(u, v) \tilde{H}_{k-1}^{*}(u, v)}{\left|\hat{H}_{k-1}(u, v)\right|^{2}+\alpha /\left|\hat{F}_{k-1}(u, v)\right|^{2}}
\end{aligned}
$$

where $(\bullet)^{*}$ denotes complex conjugate of $(\bullet)$, tilde and hat accents denote the estimate and that with constraints imposed, respectively. Care must be taken when selecting weighting $\alpha$ as it would affect the reliability of restoration. Although this technique is robust against noise, thanks to the Weiner-like filter property, but its uniqueness and convergence are greatly affected by the initial estimate of true image.

There have been other extensions of IBD in literature. For instance, Chany, Yipy, and Parky [25] ran joint optimization of deconvolution and inpainting in spatial domain to restore a degraded image with missing region. With this technique, ringing artefacts around the occlusion boundary were reduced. Zhulina [26] focused on astronomical image and extended [13] by used multichannel scheme based on maximum-likelihood of distortion hypotheses. Apart from PSF being positive, they made neither restriction on the true image nor any other PSF property. Since no input parameters were needed, the iteration was terminated by a predefined quality measure. Following multi-frame approach, short exposure atmosphere turbulence images were restored [27]. A low-pass filter was incorporated into the asymmetric multiplicative iteration to restore blurred image while removing additive noise. A more recent attempt [28] combined Mumford \& Shah piecewise-smooth image model and the sparse PSF prior regularizations. This iterative progressive restoring scheme had better rate of convergence and restoration quality.

\section{NAS-RIF}

NAS-RIF method was introduced in [14] to overcome the instability problem associated with IBD. NAS-RIF makes the same assumption on the true image as IBD, but imposes only absolutely summable, i.e., $\left(\sum\|\mathbf{h}\|<\infty\right)$, and being invertible, i.e., $\mathbf{h}^{-1}$ exists, assumptions on the PSF (Finite Impulse Response: FIR). A generic NAS-RIF is summarized in Fig 2. 


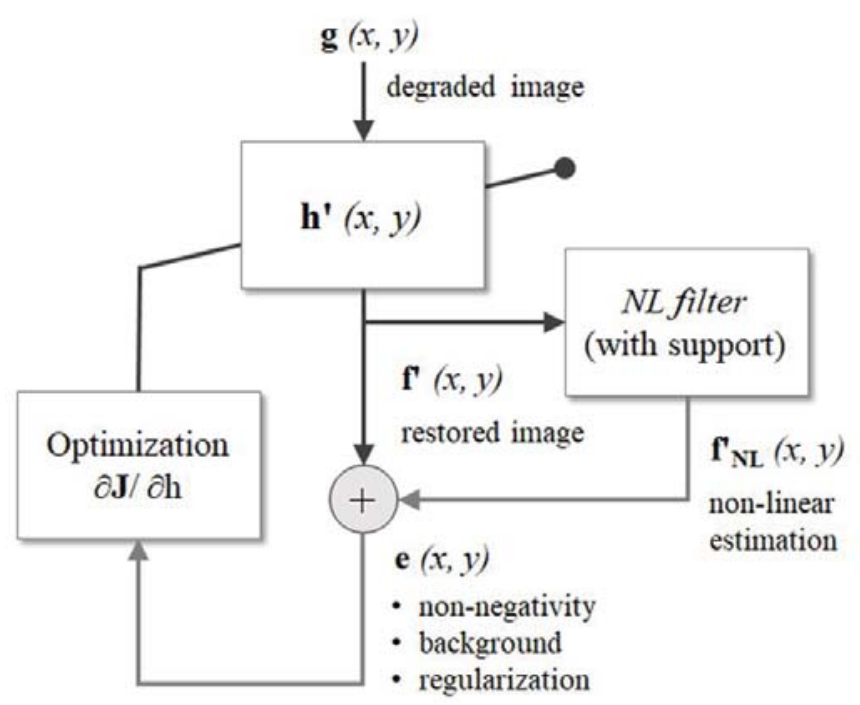

Figure 2: Diagram summarizing generic NAS-RIF algorithm

Given a degraded image $\mathbf{g}$, NAS-RIF iteratively adjusts a variable FIR filter $\mathbf{h}$, the output of this filter, $\mathbf{f}$, represents an intermediate estimation of the true image. To ensure non-negativity and supports constraints, f' is fed into a non-linear $(\mathrm{NL})$ filter, by which it is projected by using a non-expansive mapping into a space representing said constraints, $\mathbf{f}^{\prime}{ }_{\mathrm{NL}}$. The difference (error) between the estimate and its NL projection was then used to drive FIR optimization, until convergence. The NAS-RIF cost function is expressed in equation (4).

$$
J(h)=J_{\text {Dsup }}+J_{\overline{D S u p}}+J_{\text {reg }}
$$

where non-negativity, (outside) support, and regularization terms are defined in equations (5), (6), and (7) respectively.

$$
\begin{aligned}
& J_{D \text { sup }}=\sum_{(x, y) \in D \text { sup }} \hat{f}^{2}(x, y)\left[\frac{1-\operatorname{sgn}(\hat{f}(x, y))}{2}\right] \\
& J_{\overline{\text { Dsup }}}=\sum_{(x, y) \in \overline{D s u p}}\left[\hat{f}(x, y)-L_{B}\right]^{2} \\
& J_{\text {reg }}=\gamma\left[\sum_{\forall(x, y)} u(x, y)-1\right]^{2}
\end{aligned}
$$

where Dsup and $\overline{D s u p}$ are sets of pixels inside and outside the support region and LB is the background intensity.

Over decades, generic NAS-RIF has undergone several improvements and extension [17-23]. A few years after it was introduced, NAS-RIF was enhanced by multiplying a DC gain of the FIR [17] to background value in the regularization, to ensure proper scaling during optimization. In addition, to aid convergence of the non-quadratic nature of the objective function, the conjugate gradient was reset and replaced with a steepest gradient descent every fixed number of iterations. Finally, a simple threshold-based segmentation was used to better define support region, as opposed to a simple rectangle.
Kundur et al extended NAS-RIF and proposed a fusion-based approaches, to simultaneously perform deblurring by using NAS-RIF and classification by using Markov Random Field (MRF) [18]. To improve its robustness, a study [19] proposed modifying original cost function and incorporated an inter-band prediction. By using Haar wavelet decomposition, only a lower sub-band $\left(\mathrm{LL}_{0}\right)$ was restored with NAS-RIF, while the true image was then recovered from inter-band prediction. Accordingly, source of instability residing at high frequencies was neglected from the optimization. Another enhancement was also proposed [20], where Curvlet denoising was used to weaken the image noise. In that study, reliable object support and reconstruction were developed, and a logarithm function was added to speed-up the convergence and improve stability. On noise removal prior to NAS-RIF process, Siddhichai and Chambers [21] proposed a Discrete Wavelet Transform (DWT) applying to the degraded image. The squared difference between the denoised and the true images was incorporated into NAS-RIF objective function. Similar suite was pursued in [22], where lifting wavelet was used as denoising. Finally, a set of space adaptive weights were augmented into the original NAS-RIF cost function [23]. The first weight, w1, emphasizes data fidelity near the sharp edge and is multiplied with the non-negativity constrain, whilst the second weight, w2, emphasizes smoothness in presence of noise and is multiplied with the support region constrain. These weights were calculated based on noise and image intensity variances.

\section{Structural Adaptive Anisotropic Filtering}

It is evident from the existing NAS-RIF literature that noise reduction and underlying pixel distributions play an essential part in NAS-RIF stability and restoration results. This section therefore is dedicated to an effective method that has widely been applied not only in characterizing local image structure but also in constructing a non-linear filter that could preserve image feature. Structural Adaptive Anisotropic (SAA) filter was first proposed by Yang et al [29]. With this method, the extent and shape of an FIR is adapted according to underlying intensity structure. Specifically, a kernel $h(\mathbf{p})$ centered at $\mathbf{p}_{\mathbf{0}}$ is expressed in equation (8).

$$
h(\mathbf{p})=\rho\left(\mathbf{p}-\mathbf{p}_{0}\right) e^{-\left(\frac{\left[\left(\mathbf{p}-\mathbf{p}_{0}\right) \cdot n_{p}\right]^{2}}{\sigma_{p}^{2}\left(\mathbf{p}_{0}\right)}+\frac{\left[\left(\mathbf{p}-\mathbf{p}_{0}\right) \cdot n_{T}\right]^{2}}{\sigma_{T}^{2}\left(\mathbf{p}_{0}\right)}\right)}
$$

where $\rho(\mathbf{x})$ is a positive and rotationally symmetric cutoff function that satisfies the condition $\rho(\mathbf{x})=1$ when $\|\mathbf{x}\|$ is less than a maximum support radius, $r$, of the kernel. The vectors $\mathbf{n}_{\mathrm{P}}$ and $\mathbf{n}_{\mathrm{T}}$ are orthogonal unit vectors and $\mathbf{n}_{\mathrm{P}}$ is in parallel with the principal axis of oriented edge pattern. It was computed from the second moment of a mass distribution \|F (P) $\|^{2} \mathrm{dP}$ in the Fourier domain. The extents of the kernel are given by $\sigma_{\mathrm{P}}$ and $\sigma_{\mathrm{T}}$ at location $\mathbf{p}_{0}$, which are computed from corner strength, $c$, and anisotropic measure, $g$, as follow. 
$\sigma_{P}\left(\mathbf{p}_{0}\right)=\frac{r}{1+c\left(\mathbf{p}_{0}\right) / \infty}$

$\sigma_{T}\left(\mathbf{p}_{0}\right)=\left(1-g\left(\mathbf{p}_{0}\right)\right) \sigma_{P}\left(\mathbf{p}_{0}\right)$

where $\alpha$ is a normalized factor that controls how faithfully the corners and junctions should be preserved. Anisotropic and corner measures are computed from the first derivatives of the kernel center, that is,

$$
\begin{aligned}
& g\left(\mathbf{p}_{0}\right)=\frac{\left\{\iint f_{x}^{2}-f_{y}^{2} d x d y\right\}^{2}+\left\{\iint 2 f_{x} f_{y} d x d y\right\}^{2}}{\left\{\iint f_{x}^{2}+f_{y}^{2} d x d y\right\}^{2}} \\
& c\left(\mathbf{p}_{0}\right)=\left(1-g\left(\mathbf{p}_{0}\right)\right)\left|\nabla f\left(\mathbf{p}_{0}\right)\right|^{2}
\end{aligned}
$$

where $\Omega$ is the set of spatial coordinates contained by the kernel. A much accurate corner detector could be used [30] but it would be not so effective under noisy condition. It is worth noting here, that the anisotropic metric (g) preserves imaging structures via gradients and their moments, while disregarding noise by local aggregation. The resultant orientation pattern thus robustly represents local structure of image appearance, which in turn plays a major role in preserving image characteristics, pertinent to typical analyses, e.g., segmentation, edge detection, and fusion [3-6].

\section{Otsu Segmentation}

Another crucial component in NAS-RIF is initiation of the support region. It was proposed that accurate segmentation of medical object prior to optimization accelerates convergence and enhances NAS-RIF stability. Otsu method [31] is robust and versatile [32] for object from background extraction. It is based on recursively finding threshold, $t$, by minimizing the weighted within-class variance $\sigma_{w}$, i.e.,

$$
\sigma_{W}(t)=q_{1}(t) \sigma_{1}^{2}(t)+q_{1}(t) \sigma_{1}^{2}(t)
$$

where $\sigma_{1}$ and $\sigma_{2}$ are background and object class standard deviations, and $q_{1}$ and $q_{2}$ are the respective class probabilities, computed from respective pixel distributions, separated by $t$.

\section{Proposed Method}

Inspired by the preceding NAS-RIF developments [17, $19,23]$. This study proposed an enhancement to the original NAS-RIF, as shown in Fig. 3. It consists of accurate support extraction and locally weighting, obtained from structural pattern.

Conventionally, it is assumed that object is contained in a rectangular region of interest (ROI) [16]. Ong [17] has shown that for medical images, background within this ROI would be wrongly classified as object pixels, degrading the quality of restoration. This study therefore proposed a more accurate ROI or

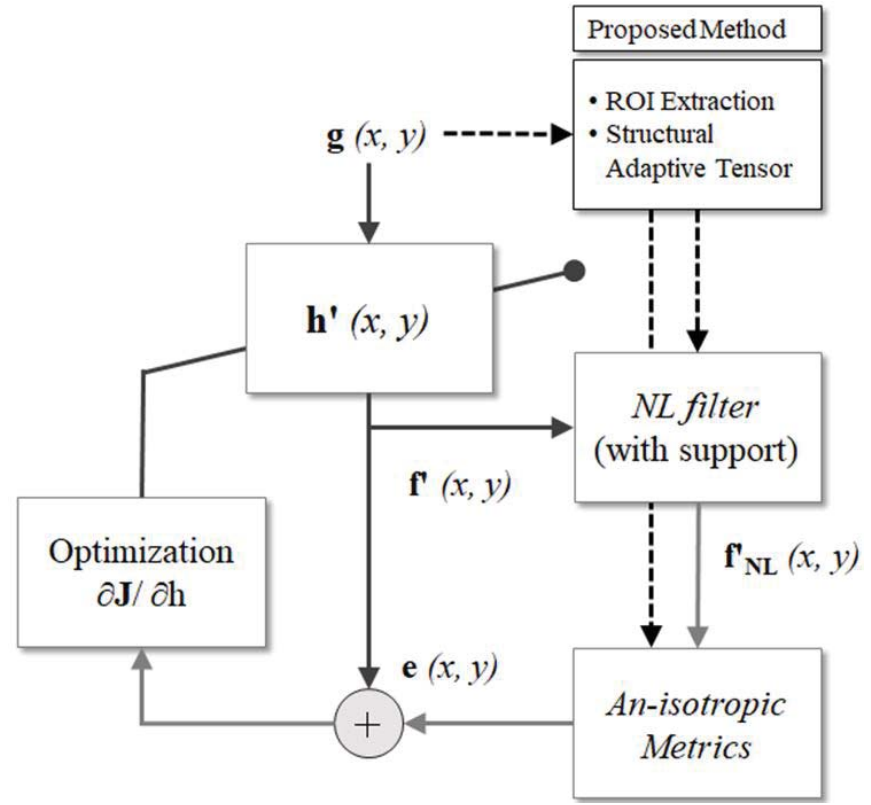

Figure 3: Diagram summarizing the proposed structural adaptive anisotropic NAS-RIF algorithm

support identification based on Otsu algorithm. Assumed that in medical acquisition, an anatomical object is located in the center of field of view (FOV), surrounded by background with a constant intensity. In this study, automatic histogram-based segmentation thus sufficed. The extracted ROI(s) was defined as binary mask (1 and 0 ), corresponding to pixels within and outside support region respectively.

Several NAS-RIF studies also demonstrated that image noise causes substantial instability, thus undermining the effectiveness of the deconvolution. Attempts have been made by empirically choosing low frequency sub-band signal, where noise effect was remedied or denoising input images, prior to the main process. However, it was later demonstrated that contributions to energy minimization from involving pixels can be adjusted by a weighting factor, calculated from underlying image pattern [23]. In this study, noise amplification was hence regularized by the structural anisotropic measure as given in Equation (10). Further speed up was achieved by pre-calculating the anisotropic map prior to entering the main loop. The weight characterizing the data fidelity, neglecting noise distribution due to spectral second moment, was incorporated into non-negativity cost. Moreover, unlike [23] another separate balancing factor, emphasizing smoothness was not needed. Specifically, the cost functions (5) and (6) were therefore modified as follow:

$$
\begin{aligned}
& J_{D \text { sup }}=\sum_{(x, y) \in D \text { sup }} w(x, y) \hat{f}^{2}(x, y)\left[\frac{1-\operatorname{sgn}(\hat{f}(x, y))}{2}\right] \\
& J_{\overline{D \text { sup }}}=\sum_{(x, y) \in \overline{D \text { sup }}}\left[\hat{f}(x, y)-\propto L_{B}\right]^{2} \\
& J_{\text {reg }}=\sum_{(x, y)} \nabla^{2} \hat{f}(x, y)+\gamma\left[\sum_{\forall(x, y)} u(x, y)-1\right]^{2}
\end{aligned}
$$


where the inversed anisotropic measure was denoted as $w(\mathrm{x}, \mathrm{y})=1-g(\mathrm{x}, \mathrm{y})$ in Equation (12) to avoid an ambiguity with degraded input image, $g$. Regularize parameters, $\alpha$ and $\gamma$, were also added to allow for non-unity DC (Direct Current) gain compensation, when the image has non-zero background, and to prevent trivial zero of an estimated PSF, during optimization, respectively. Without loss of generalization ability, the values were set to 1.0 as suggested by [17]. A Laplacian of image was also incorporated into regularization term to further reduce noise amplification [23]. The remaining components of NAS-RIF, including the calculation of cost function derivative and optimization procedure followed the original proposal [14].

\section{EXPERIMENTAL RESULTS}

This section provides visual and numerical assessments of the proposed extension of NAS-RIF restoration. Specifically, they are degraded images by predefined Gaussian PSF and noise, support region $\left(d_{\text {sup }}\right)$ extraction, recursive restoration process, image quality assessment (both visually and numerically), and convergence analysis.

The experiment was carried out on medical images from public repositories. The proposed NAS-RIF algorithm was implemented in C\# language by using Visual Studio ${ }^{\circledR}$ by Microsoft ${ }^{\mathrm{TM}}$ Cooperation. Other trivial image processing algorithms, such as linear operations, convolution, and display was based on Emgu CV library [33] ver. 3.4. Three-dimensional graphical representation was programmed using OpenGL ${ }^{\circledR}$ by Krosnos ${ }^{\mathrm{TM}} \mathrm{Group}$. For more detailed account on the NAS-RIF algorithm, readers are referred to the pseudo-code listed in the Appendix or the original proposals $[14,15,16]$.

\section{Degraded Images}

In the following experiments each image was convoluted with a Gaussian PSF with known standard deviations, which were 5 and 7 pixels, expressed with kernel sizes of 11 and 15 pixels, respectively.

$$
h(\mathbf{p})=\frac{1}{\sigma \sqrt{2 \pi}} \exp \left(\frac{\mathbf{p}^{2}}{2 \sigma^{2}}\right)
$$

Selected images and degraded version are shown in Fig. 4.

\section{Support Region Extraction}

Support region $\left(\mathrm{d}_{\text {sup }}\right.$ ) plays a crucial part in NAS-RIF. It was shown in previous studies that accurate support region could improve its stability and performance. This paper thus adopted an automatic Otsu segmentation to obtain the initial ROI masking for Eq. (12) and (13). The extraction results on two sample images are illustrated in Fig. 5.

It is evident from the figure that for medical images, Otsu algorithm could efficiently and robustly extract the support ROI for this purpose. It should be noted however, that exact delineation of anatomical boundary was not required in NAS-RIF, hence minor over- or under-segmented pixels would be overwhelmed by correctly segmented majority.

\section{Structural Adaptive Anisotropic Measure}

The key ingredient of the proposed method is the recognition of anisotropic pattern, characterizing local image orientation. Fig. 6 shows three examples of different orientation patterns. They are right and left orientations and isotropic region with no apparent structure.

Fig. 7 illustrates two examples of anisotropic measures (g) of high (top) and low (bottom) noise images. Note that the brighter values correspond to highly structured patterns and therefore higher fidelity, and vice versa.

It is evident that regardless of PSF extents, the anisotropic function offered consistent characterization of data fidelity. It is also robust against noise (top row). Specifically, recovered pattern reflects its appearance. Although some background noise was also noticeable in the top
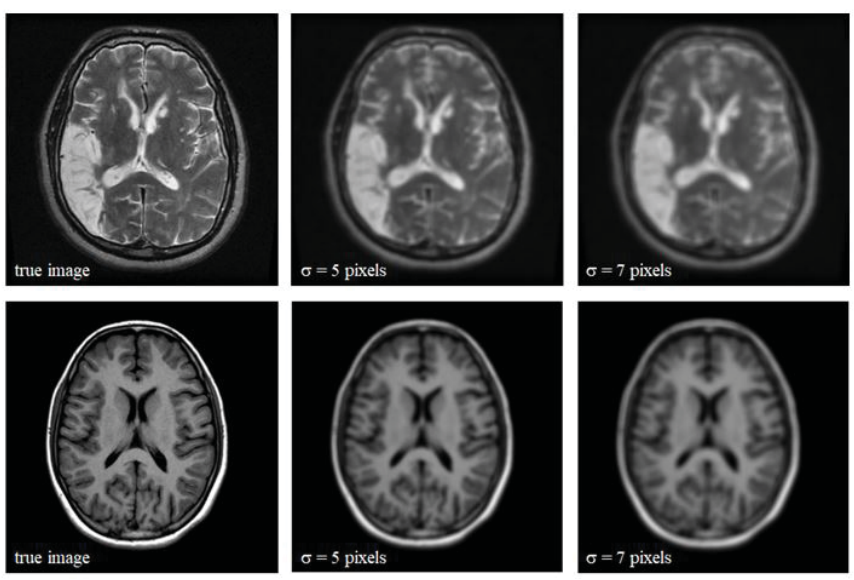

Figure 4: Original sample images (left) and that degraded with 5 (middle) and 7 (right) pixels standard deviation Gaussian kernels
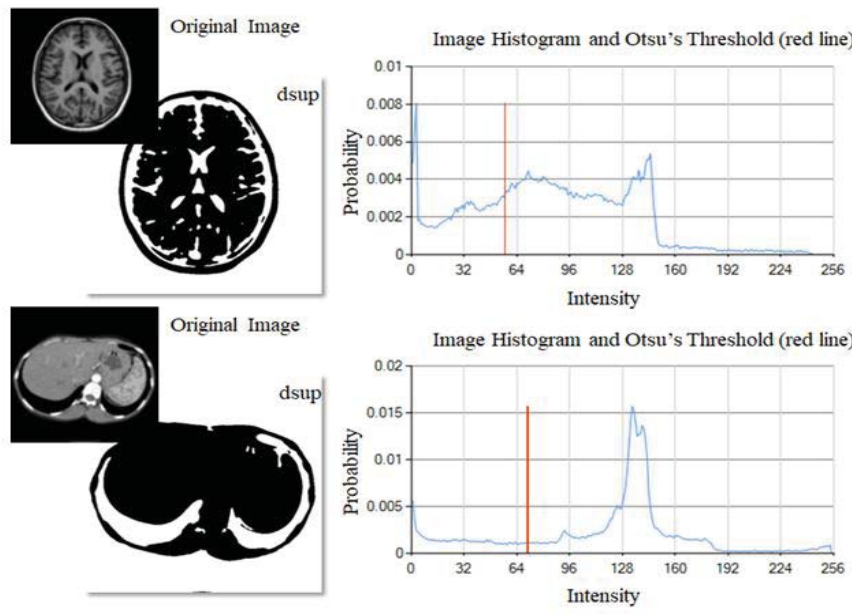

Figure 5: Original sample images (insets), NAS-RIF supporting regions (dsup) (binary) and their intensity distributions (graph) with respective Otsu's threshold values (red-line) 


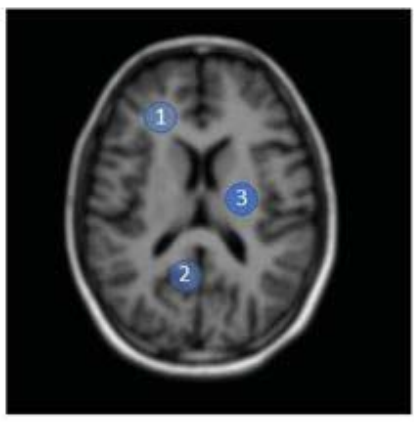

(1) Right orientation pattern

(2) Strong left orientation pattern

(3) Isotropic pattern (low g)

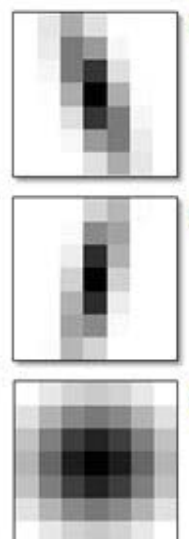

(1)

$\mathrm{g}=0.7015$

$\mathrm{c}=0.0488$

$\theta=70.768^{\circ}$

$\mathrm{g}=0.7510$

$\mathrm{c}=0.0454$

$\theta=102.962^{\circ}$

$\mathrm{g}=0.0954$

$\mathrm{c}=0.0124$

$\theta=0.8107^{\circ}$

Figure 6: An example of an image (left) with three selected regions (1) - (3) and their corresponding pattern parameters (right), i.e., isotropic measure (g), corner strength (c) and orientation angle (theta)
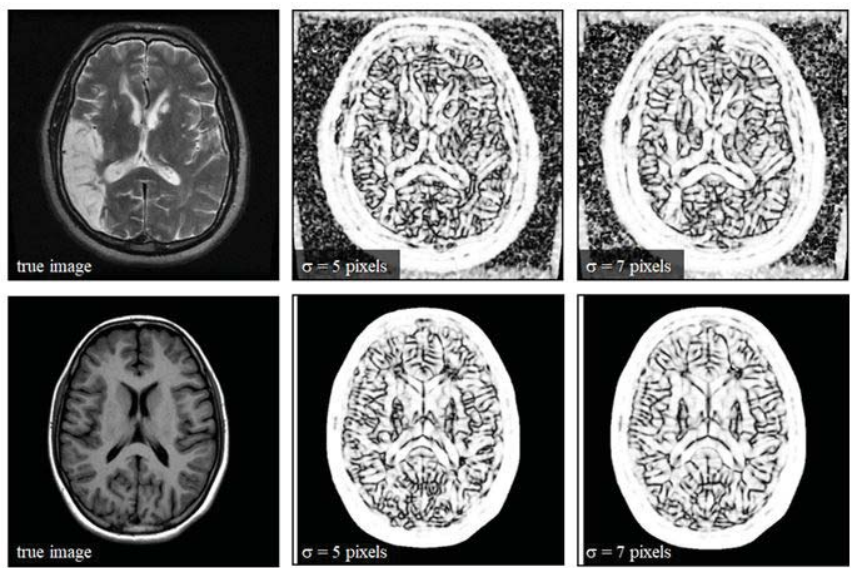

Figure 7: Original high (top) and low (bottom) noise images (left) and anisotropic measures of the corresponding images degraded with 5 (middle) and 7 (right) pixels s.d. Gaussian kernels

image, it would have been removed by $\mathrm{ROI}$ extraction by Otsu and caused slight effect (outside support) on the NAS-RIF procedure.

\section{Recursive Image Restoration}

On elucidating the merits of the proposed enhancement, assessments were carried out on both high and low noise data. Due to different acquisition protocol, varying degree of noise was exhibit in medical images. In the experiment, estimation of PSF and true images on both high and low noise cases were demonstrated. Identical NAS-RIF parameter settings as per Eq. (12)-(14) were maintained in both scenarios. Table 1 lists relevant parameters specified in this study. Note that conjugate reset refers to the number of conjugate gradient iterations before each re-calculation of the direction by means of robust steepest gradient descent, to improve convergent stability. Convergence criteria that terminated the optimization were either updates falling below $1.0 \times 10^{-4}$ (Min. Step) of kernel magnitude or reaching 1200 (Max.) iterations.
Table 1: NAS-RIF Parameter Settings

\begin{tabular}{|l|l|l|l|}
\hline $\begin{array}{l}\text { Kernel } \\
\text { Parameters }\end{array}$ & Values & $\begin{array}{l}\text { System } \\
\text { Parameters }\end{array}$ & Values \\
\hline $\begin{array}{l}\text { Small PSF } \\
\text { size }\end{array}$ & $11 \times 11$ & $\begin{array}{l}\text { Max. } \\
\text { Iterations }\end{array}$ & 1200 \\
\hline $\begin{array}{l}\text { Small PSF } \\
\text { s.d. }\end{array}$ & $5 \times 5$ & $\begin{array}{l}\text { Regularize } \\
(\mathrm{\gamma}, \alpha)\end{array}$ & $1.0,1.0$ \\
\hline $\begin{array}{l}\text { Large PSF } \\
\text { size }\end{array}$ & $15 \times 15$ & $\begin{array}{l}\text { Max step } \\
(\Delta \text { hi,j })\end{array}$ & $1.0 /\left\|h^{\prime}\right\|$ \\
\hline $\begin{array}{l}\text { Large PSF } \\
\text { s.d. }\end{array}$ & $7 \times 7$ & $\begin{array}{l}\text { Min step } \\
(\Delta \text { hi,j })\end{array}$ & $1.0 \mathrm{E}-4 /\left\|h^{\prime}\right\|$ \\
\hline $\begin{array}{l}\text { PSF kernel } \\
\text { size (h') }\end{array}$ & $7 \times 7$ & $\begin{array}{l}\text { Conjugate } \\
\text { reset }\end{array}$ & 1 at every 20 \\
\hline
\end{tabular}
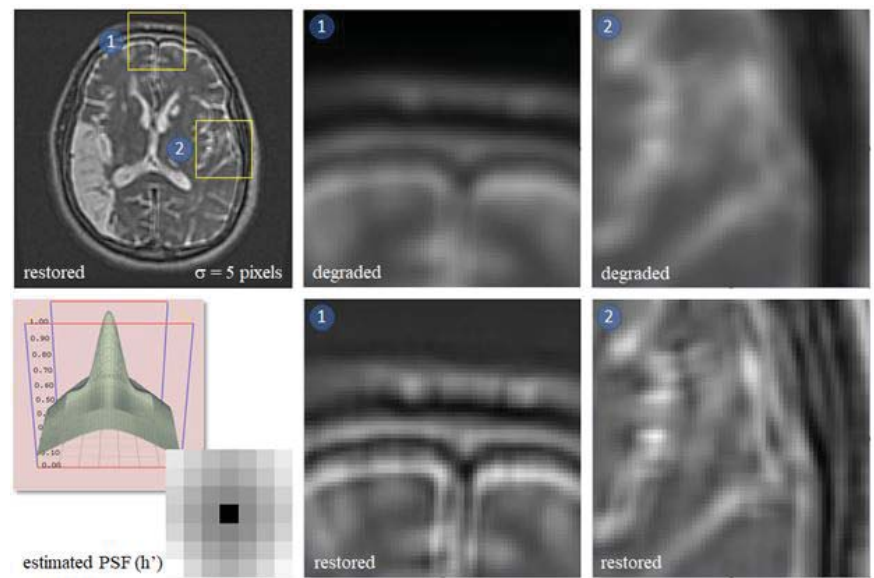

Figure 8: Restored noisy image (top left) and comparison in selected zoomed-in regions (middle and right) between degraded with small PSF (top) and restored (bottom) images. Bottom left inset represents restoring PSF

\section{Visual Quality Assessment}

A low noise image was undergone both small (5 pixel) and large (7 pixels) $\sigma$-Gaussian PSFs (Eq. 15), as shown in Fig 4 (top row). Fig. 8 (top) and (bottom) illustrates restored images and comparison in selected zoomed-in regions (labelled as 1 and 2). Degraded with small PSF, it is evident that oriented edge structure and gray and white matter boundaries were mostly recovered. Bottom left inset represents restoring PSF in 3 - and 2 dimensions.

Similarly, Figure 9 illustrates a zoomed-in region (labeled as 2) of a degraded image with a large PSF (middle) and restored image (right), by using the estimated PSF (left inset).

The same experiment was carried out on a low noise image, as shown in Fig 4 (bottom row). The estimated PSF in both small (top left) and large (bottom left) standard deviation in 3- and 2 dimensions are illustrated in the insets. The middle and right images, show degraded, with 5 (top) and 7 (bottom) pixels $\sigma$, and the corresponding 

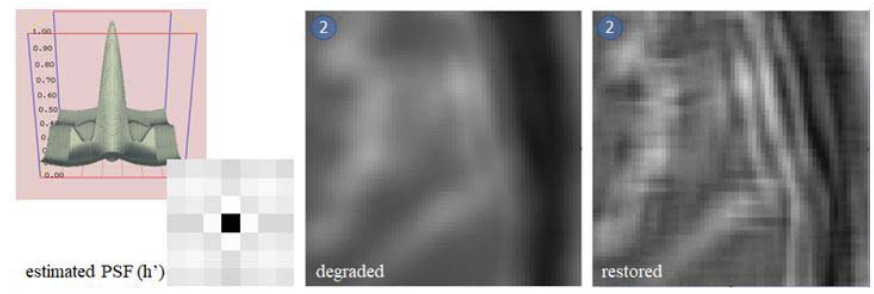

Figure 9: Restored noisy image (top left) and comparison in selected zoomed-out region (middle and right) between degraded with large PSF (top) and restored (bottom) image. Left inset represents restoring PSF

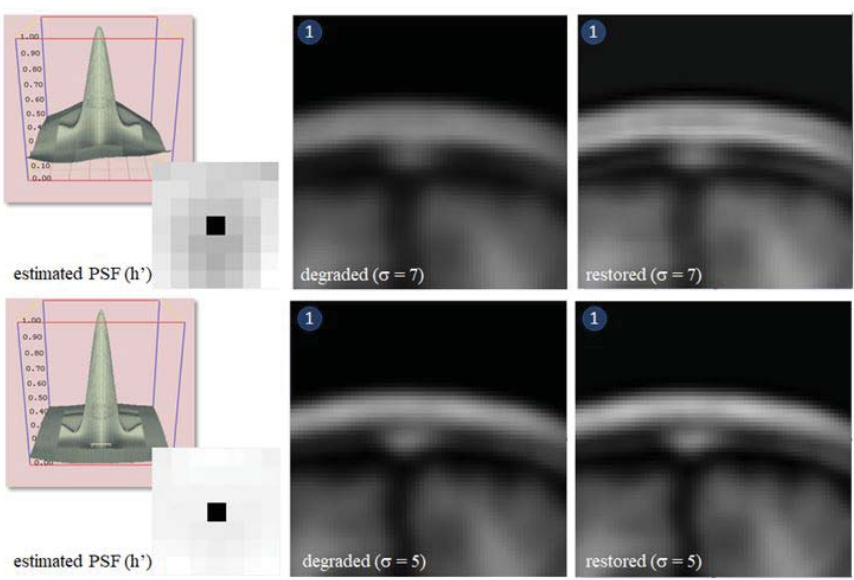

Figure 10: Estimated restoring PSF (left) and comparison in a zoomed-in frontal regions (middle) and the corresponding restored images (right), after degraded with small (top) and large (bottom) blurs

restored images, respectively. It can be seen from the Fig. 10 that, for the selected frontal region, boundaries of connective tissue were clearly restored.

\section{Quantitative Fidelity Evaluations}

Evaluation of image fidelity depends on properties of interest. Since medical imaging typically involves basic analyses, namely region segmentation, boundary detection, and fusion, relevant properties were thus evaluated. Firstly, definition of object boundary is characterized by sharpness and clarity of the image. The first metrics was edge strength (EDGE). It was defined by an averaged gradient magnitude, $\left\|\nabla f^{*}\right\|$, over the support region $\left(d_{\text {sup }}\right)$. For a more general perception system, SSIM (Structural SIMilarity) [34] was employed, for its strong relation between computerized imaging and human visual neurobiology. It was expressed by a (uniform) weighted combination between luminance, contrast, and structural appearance similarities between true and restored images. Likewise, the value was calculated over $\mathrm{d}_{\text {sup }}$. Its expression, comparing images $\mathbf{x}$ and $\mathbf{y}$, is given in Eq (16).

$\operatorname{SSIM}(\mathbf{x}, \mathbf{y})=\frac{\left(2 \mu_{x} \mu_{y}+\epsilon\right)\left(2 \sigma_{x y}+\epsilon\right)}{\left(\mu_{x}^{2}+\mu_{y}^{2}+\epsilon\right)\left(\sigma_{x}^{2}+\sigma_{y}^{2}+\epsilon\right)}$ where $\mu_{x}, \mu_{y^{\prime}} \sigma^{2}{ }_{x}, \sigma_{y^{\prime}}^{2}$ and $\sigma_{x y}$ are, respectively, averaged intensities of images $x$ and $y$, their variances and covariance. $\epsilon$ is a constant stabilizing the division by small dominator. Finally, to determine if the proposed restoration would benefit image fusion, NCC (Normalized Cross-Correlation) [4] between true and restored images was also evaluated.

Fig. 11 depicts EDGE, SSIM, and NCC of the noisy image, underwent small (a) and large (b) kernels degradation, respectively, during the restoration process. Likewise, Fig. 12 depicts the same evaluations for the low noise image.

\section{Convergence Analysis}

On analyzing convergence behavior of the proposed method, Peak Signal to Noise Ratio (PSNR) of the restored against true image was measured based on PSNR $=10 \log _{10}\left(\right.$ error $^{2} /$ peak $)$ of pixels within the sup

a)

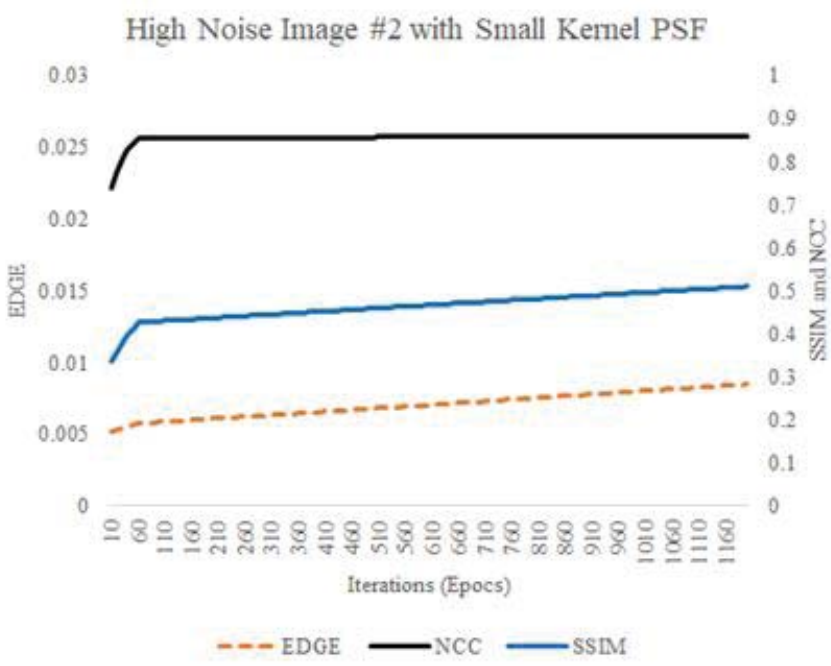

Figure 11a: Quantitative fidelity evaluation for the noisy image given small PSF

b)
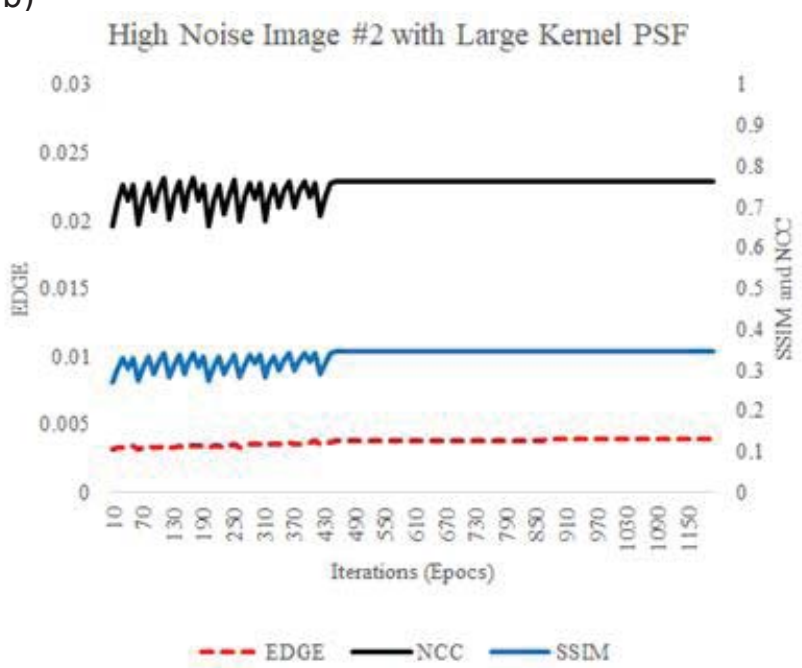

Figure 11b: Quantitative fidelity evaluation for the noisy image given large PSF 
a)

Low Noise Image \#1 with Small Kernel PSF

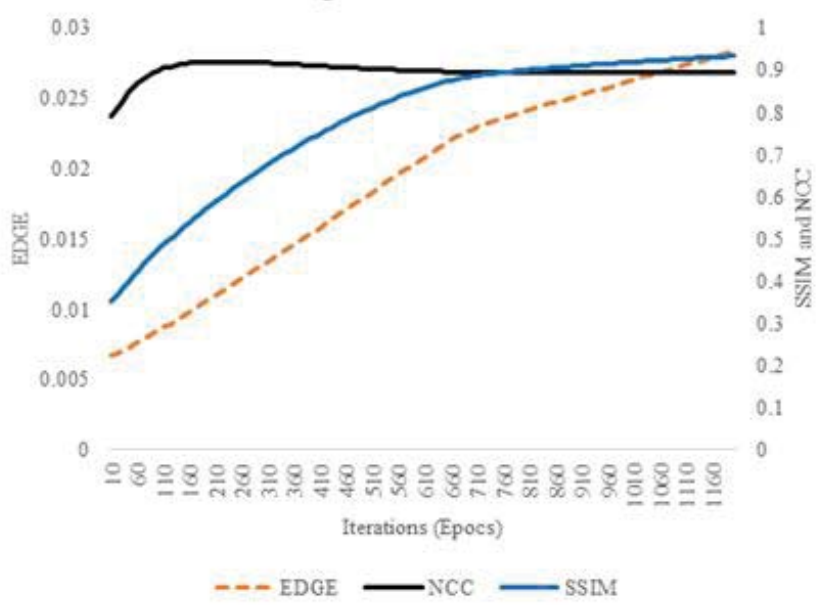

Figure 12a: Quantitative fidelity evaluation for the low noise image given small PSF

Low Noise Image \#1 with Large Kernel PSF

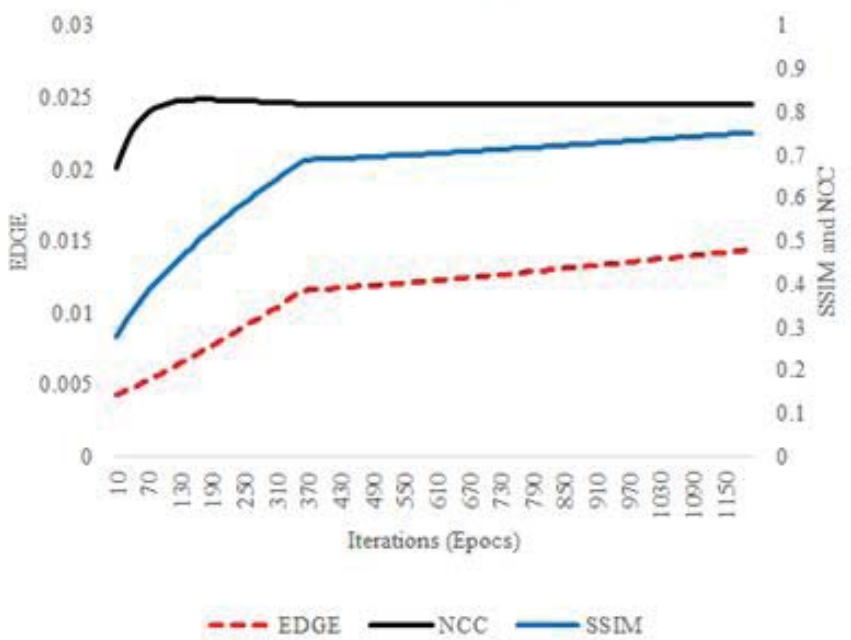

Figure 12b: Quantitative fidelity evaluation for the low noise image given large PSF

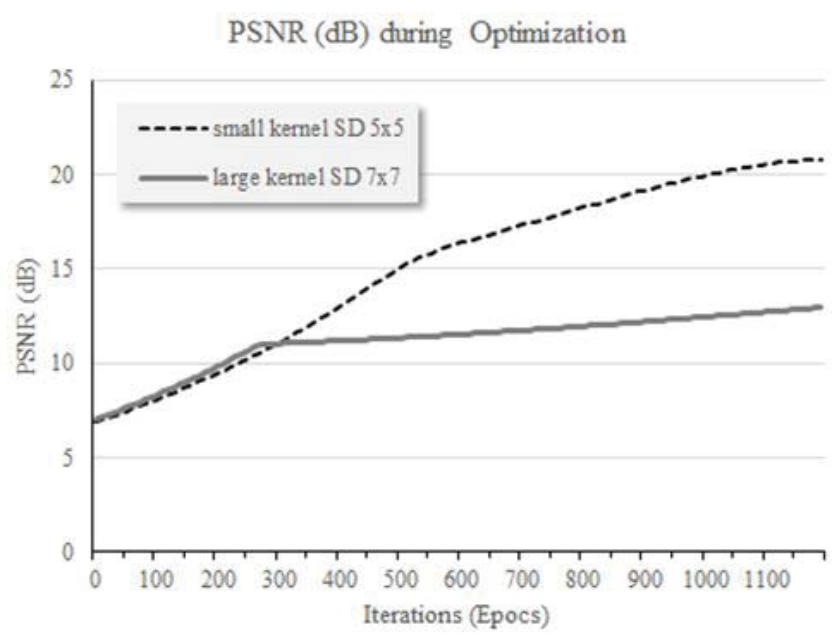

Figure 14a: PSNR (dB) of the low noise image given small and large PSF a)

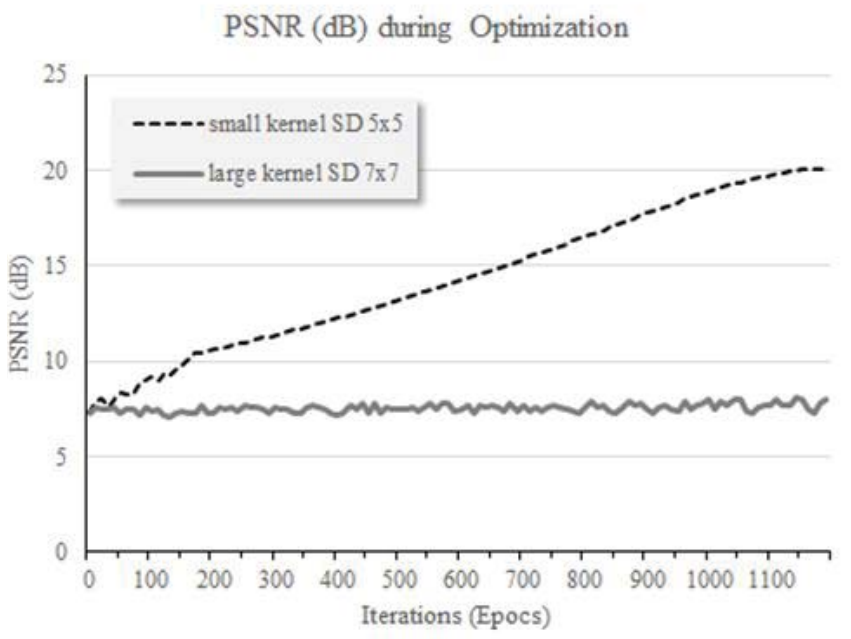

Figure 13a: PSNR (dB) of the noisy image given small and large $P S F$

b)

Relative Kernel Update $(\mathrm{dB})$ during Optimization

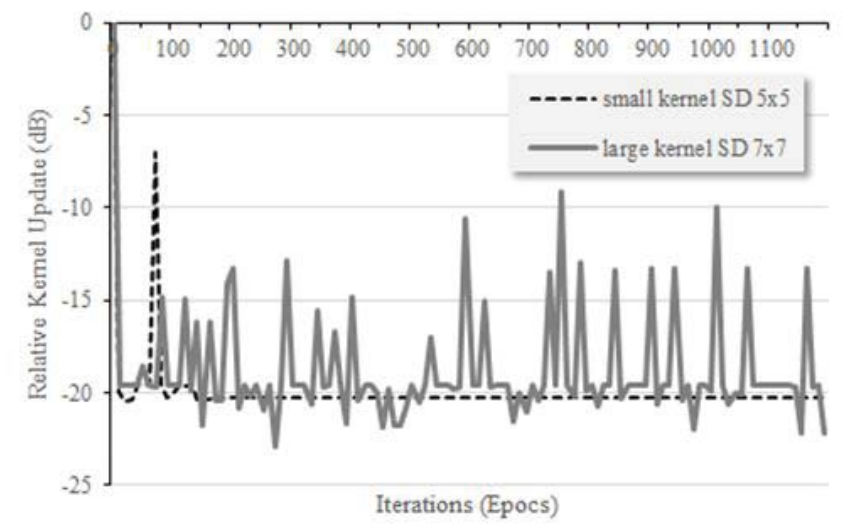

Figure 13b: Relative kernel updates ( $d B)$ of the noisy image given small and large PSF

Relative Kernel Update (dB) during Optimization

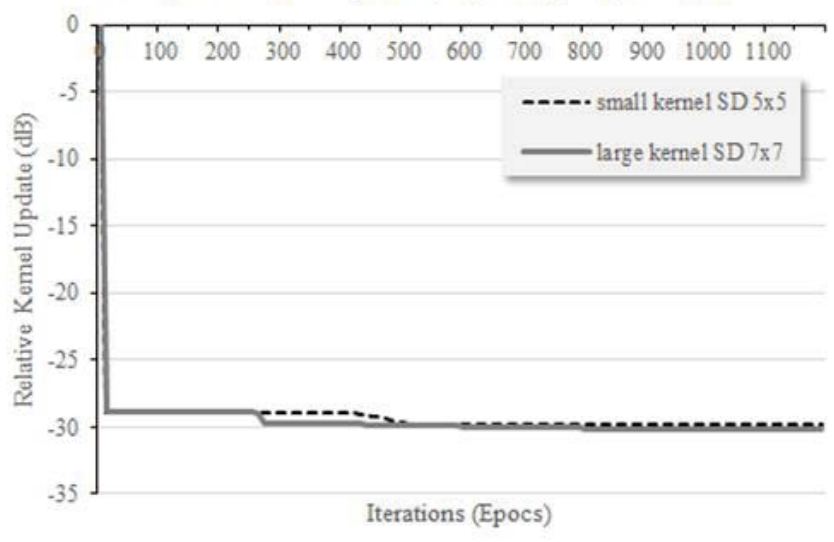

Figure 14b: Relative kernel updates $(d B)$ of the low noise image given small and large PSF 
port region. The relative update of estimated (restoring) PSF was calculated by Update $(\mathrm{dB})=10 \log _{10}\left(\Sigma\left|\Delta \mathrm{h}_{k}\right| /\right.$ $\left.\Sigma\left|\Delta \mathrm{h}_{1}\right|\right)$, where $\mathrm{k}$ was the iteration index. For the noisy image, the PSNR and relative kernel update are shown in Fig. 13 ( $a$ and $b$, respectively). The dotted and gray lines represent results for images degraded with small and large blur, respectively. The same experiment was repeated in a low noise case and corresponding results are shown in Fig. 14.

\section{DISCUSSION}

Visual assessments (Fig. 8-10) confirmed that enhanced NAS-RIF could recovered degraded features in both low and high noise cases. Especially, gray and white matter separation and brain boundary became more apparent. Importantly, the estimated restoring PSF in highly blurred images resembled sharpening, i.e., a reciprocal filter of the degrading PSF. In less severely blur, however, the results differed between low and high noise images. In the former case, the estimated PSF remained a sharpening with lesser degree, whilst in the latter case, the kernel was also elevated by a quadratic function to compensate not only for blur but also for inherent noise.

To investigate the adaptability of the proposed NAS-RIF variation quantitatively, three accuracy and fidelity metrics related to biomedical imaging tasks were evaluated. In the noisy image (Fig. 11), the EDGE strength increased as it proceeded, although at slightly higher rate in small PSF case (a) than in the large one (b).For a general-purpose vision system, SSIM, which characterizes not only image luminance and contrast restoration but also its structural appearance, was evaluated. The value consistently was improved but at decreasing rate toward the end for the small PSF case (a). Fluctuation at 0.3 was found in the large PSF case (b), during approximately the first 450 iterations, after which it converged. Similar characteristics were established for the NCC in both cases. For the lower noise image (Fig. 12), the improvements on these fidelity metrics were more pronounced in both small (a) and large kernel (b) cases. Convergences of EDGE and NCC in the latter case, nonetheless, started earlier (350 iterations) than did the former one (700 iterations).

On convergence inspection, if noisy image (Fig. 13) was degraded with low level blur, kernel update started to decay since the first few hundred iterations and PSNR did stabilized but at later stage. At higher level blur, small kernel update began early but fluctuated within -23 to -30 $\mathrm{dB}$, while PSNR had not much been improved, in which case, the procedure was terminated when maximum number of iterations was reached. For a lower noise case (Fig. 14), similar visual results were established. Numerically, however, both PSNR and kernel updates had better convergence behavior, in which case, small incremental kernel updates relative to its magnitude criterion was met upon termination.

\section{CONCLUSION}

Image enhancement is the first and important step toward various machine learning algorithms for Computer Aided Diagnosis (CAD) and Interventional (CAI) [35]. NAS-RIF and its variants were proved a viable tool in recovering the true from degraded image. The technique was based on iterative blind image deconvolution scheme [13] and was first proposed by Kundur and Hatzinakos [14]. Original NAS-RIF was found efficient and suitable for medical and astronomical images. Thus far, studies have shown that support region and multi-resolution analyses, and objective regularization could be implemented to further improve NAS-RIF stability and its convergence behavior. This study adopted this paradigm and proposes multi-modal automatic segmentation to define more accurate support and introduces a structural anisotropic weight, corresponding to image fidelity but was more robust against intensity noise, to adaptively tune the non-negativity cost. The experimental results reported herein demonstrated that the enhanced NAS-RIF could restored medical images with low and high inherent noise that were degraded with PSF with varying kernel sizes.

\section{ACKNOWLEDGEMENT}

The authors would like to thank various public DICOM repositories, e.g., www.dicomlibrary.com/, www.aycan. $\mathrm{de} /$, and the referenced url to imaging database therein for images used in the above experiments. This study was partly supported by SUT-OROG grant.

\section{APPENDIX}

Table 2: Summary of the Proposed Enhanced NAS-RIF

\begin{tabular}{|c|c|c|}
\hline Line & Operations & Remarks \\
\hline 1: & input: $g(x, y)$ & degraded image \\
\hline 2: & initialize FIR $h(x, y)$ as unit impulse & \\
\hline 3: & compute anisotropic map of $\mathrm{g}(\mathrm{x}, \mathrm{y})$ & eq. (10) \\
\hline 4: & segmentation of support ROI & eq. (11) \\
\hline 5: & for $\mathrm{k}=0$ to $\max \mathrm{kdo}$ & main iterations \\
\hline 6: & compute estimated $f^{\prime}(x, y)$ & $(\mathrm{hk} * \mathrm{~g})(\mathrm{x}, \mathrm{y})$ \\
\hline 7: & compute Laplacian of $f^{\prime}(x, y)$ & Ref. [23] \\
\hline 8: & compute convex projection $\mathrm{f}^{\prime} \mathrm{NL}(\mathrm{x}, \mathrm{y})$ & based on ROI \\
\hline 9: & minimization routine & conj. gradient \\
\hline 10: & compute gradient $\partial \mathrm{J} / \partial \mathrm{h}$ & \\
\hline 11: & find conjugate gradient direction & $\mathrm{dk}$ \\
\hline 12: & update $\mathrm{hk}+1=\mathrm{hk}+\beta \mathrm{dk}$ & golden section \\
\hline 13: & check for convergence & no update on $\mathrm{h}$ \\
\hline 14: & endfor & $\mathrm{f}^{\prime}=(\mathrm{hk} * \mathrm{~g})$ \\
\hline 15: & end NAS-RIF & \\
\hline
\end{tabular}




\section{REFERENCES}

1. Chen, T.J., Chuang, K.S., Chang, J.H., Shiao, Y.H., Chuang, C.C., 2006. A blurring index for medical images. Journal of Digital Imaging. 19(2), 118-125.

2. van Overveld, I. M. C. J., 1995. Contrast, noise, and blur affect performance and appreciation of digital radiographs. Journal of Digital Imaging. 8(4), 68-179.

3. Panyavaraporn, J., Horkaew, P., 2018. Classification of Alzheimer's disease in PET scans using MFCC and SVM. International Journal on Advanced Science, Engineering and Information Technology, 8(5), pp. 1829-1835.

4. Khaminkure, A., Horkaew, P., Panyavaraporn, J., 2017. Building a brain atlas based on gabor texture features, Proc. 14th Intl. Conference on Computer Science and Software Engineering, JCSSE 2017, 8025935, pp. 1-5.

5. Ipatov, Y., Krevetsky, A., Andrianov, Y., Sokolov, B. [2017]. Creation of image models for evolving objects on dynamically changing scenes. Journal of Applied Engineering Science, 15(4), 540-545.

6. Ipatov, Y., Krevetsky, A., Andrianov, Y., Sokolov, B., [2017]. Robust classification of texture land forrest inventory based on model of minimally sufficient features. Journal of Applied Engineering Science, 15(3), 236-241.

7. Turner, J.N., Lasek, S., Szarowski, D.H., 2001. Confocal optical microscopy. Encyclopedia of Materials: Science and Technology (Second Edition), pp. 15041509.

8. Jannetta, A., Jackson, J.C., Kotre, C.J., Birch, I.P., Robson, K.J., Padgett, R., 2004. Mammographic image restoration using maximum entropy deconvolution, Physics in Medicine and Biology, 49(4997), 18 pages.

9. Kundur, D., Hatzinzkos, D., 1996. Blind image deconvolution. IEEE Signal Processing Magazine, 13(8), 43-64.

10. Ruiz, P., Zhou, X., Mateos, J., Molina, R., Katsaggelos, A., 2015. Variational Bayesian blind image deconvolution: a review. Digital Signal Processing. 47, 116-127.

11. Molina, R., Mateos, J., Katsaggelos, A., 2006. Blind deconvolution using a variational approach to parameter, image, and blur estimation. IEEE Trans. Image Process, 15(12), 3715-3727.

12. Bishop, T.E., Babacan, S.D., Amizic, B., KatsaggeIos, A.K., Chan, T., Molina, R., 2007. Blind image deconvolution: problem formulation and existing approaches, CRC Press, 49 pages.

13. Ayers, G. R., Dainty, J. C., 1988. Iterative blind deconvolution method and its applications, Optics Letters, 13(7), 547-549.
14. Kundur, D., Hatzinakos, D., 1995. A novel recursive filtering method for blind image restoration, Proc. IASTED International Conference on Signal and Image Processing (SIP), 428-431.

15. Kundur, D., Hatzinzkos D., 1996A. On the global asymptotic stability of the NAS-RIF algorithm, Proc. International Conference on Image Processing, 7376.

16. Kundur, D., Hatzinzkos D., 1998. A novel blind deconvolution scheme for image restoration using recursive filtering, IEEE Signal Processing Magazine, 26(2), 375-390.

17. Ong, C.A., Chambers J.A., 1999. An enhanced NAS-RIF algorithm for blind image deconvolution, IEEE Transactions on Image Processing, 8(7), 988992.

18. Kundur, D., Hatzinakos, D., Leung, H., 2000. Robust classification of blurred imagery, IEEE Transactions on Image Processing, 9, 243-255.

19. Maysuyama, M., Tanji, Y., Tanaka, M., 2000. Enhancing the ability of NAS_RIF algorithm for blind image deconvolution, Proc. IEEE International Symposium on Circuits and Systems (ISCAS). 553-556.

20. Yang, Y., Yang, Z., Shen, T., Chen, B., 2012. ENASRIF algorithm for image restoration, SPIE, Photonics Asia, 1, 85581Z-85581Z-8.

21. Siddhichai, S., Chambers, J.A., 2000. Wavelet transform-based noise reduction schemes to improve the noise sensitivity of the NAS-RIF algorithm for blind image deconvolution, Proc. Signal Processing Conference, 4 pages.

22. Wang, H., Qi, S., Liu, Y., 2009. An improved NASRIF algorithm based on the lifting scheme for blind image restoration, IEEE Circuits, Communications and Systems, 423-426.

23. Chow, T.W.S., Li, X.D., Cho, S.Y, 2000. Improved blind image restoration scheme using recurrent filtering, Proc. IEE Vision Image and Signal Processing, 147(1), 23-28.

24. Kundur, D., Hatzinakos, D., 1996B. Blind image deconvolution. IEEE Signal Processing Magazine, 13(3), 43-64.

25. Chany, T.F., Yipy, A.M., Parky, F.E., 2005. Simultaneous total variation image inpainting and blind deconvolution. International Journal of Imaging System and Technology, 15(1), 92-102.

26. Zhulina,Y.V., 2006. Multiframe blind deconvolution of heavily blurred astronomical images. Applied Optics, 45(28) 7342-7352.

27. Zhang, J., Zhang, Q., He, G., 2009. Blind deconvolution of a noisy degraded image, Applied Optics, 48(12), 2350-5. 
28. Hanocka, R., Kiryati, N., 2015. Progressive blind deconvolution. LCNS Computer Analysis of Images and Patterns, 9257, 313-325. Yang, G.Z., Burger, P., Firmin, D.N., Underwood, S.R. 1996. Structure adaptive anisotropic image filtering, Image and Vision Computing, 14, 135-145.

29. Wu, J., Feng, Z., Ren, Z., 2014. Improved structure-adaptive anisotropic filter based on a nonlinear structure tensor. Cybernetic and Information Technology, 14(1), 112-127.

30. Otsu, N., 1979. A threshold selection method from gray-level histograms. IEEE Trans. Sys., Man., Cyber. 9(1), 62-66.

31. Sezgin, M., Sankur, B., 2004. Survey over image thresholding techniques and quantitative performance evaluation. Journal of Electronic Imaging. 13(1), 146-165.

32. Shi, S., 2013. Emgu CV essentials, Packt Publishing, 118 pages.

33. Wang, Z., Bovik, A.C., Sheikh, H.R., Simoncelli, E.P., 2004. Image quality assessment: from error visibility to structural similarity. IEEE Transactions on Image Processing. 13(4), pp. 600-612.

34. Garcia-Nieto, J., Alba, E., Jourdan, L., Talbi E., 2009. Sensitivity and specificity based multiobjective approach for feature selection: Application to cancer diagnosis, Information Processing Letters, 109(16), 887-896. 